\title{
The clinical characteristics and prognostic analysis of Chinese advanced NSCLC patients based on circulating tumor DNA sequencing
}

This article was published in the following Dove Press journal: OncoTargets and Therapy

\section{Chuangzhou Rao' \\ Liangqin Nie' \\ Xiaobo Miao' \\ Yunbao $\mathrm{Xu}^{\prime}$ \\ Bing $\mathrm{Li}^{2}$ \\ Tengfei Zhang ${ }^{2}$}

'Radiotherapy \& Chemotherapy Dept 2, Ningbo No. 2 Hospital, Zhejiang, ${ }^{2}$ Burning Rock Biotech, Guangzhou, People's Republic of China
Correspondence: Chuangzhou Rao Radiotherapy \& Chemotherapy Dept 2, Ningbo No. 2 Hospital, 4I Xibei Street, Ningbo, Zhejiang 3150I0, People's Republic of China

Tel +86 I53 56884370

Fax +86 57483870612

Email raochuangzhou@।63.com
Purpose: Circulating tumor DNA (ctDNA) is a noninvasive and real-time marker for tumor diagnosis, prognosis, and prediction. However, further investigations about ctDNA prognostic and predictive value are still needed, and conclusions from several studies were inconsistent. Experimental design: We performed capture-based targeted ultradeep sequencing on liquid biopsies from a cohort of 34 advanced Chinese non-small-cell lung cancer (NSCLC) patients and analyzed the clinical use of ctDNA in this study.

Results: On the basis of clinical characteristics of the 34 NSCLC patients, we found that brain metastasis correlated with shorter progression-free survival (PFS) and is more prone to happen in younger patients. After ctDNA sequencing, we analyzed the prognostic value of baseline ctDNA. In osimertinib-treated group, high max allelic fraction (maxAF) correlated with shorter PFS. But for the cohort of 34 patients, no correlation can be observed between maxAF and PFS. We also presented two cases to demonstrate the value of disease progression prediction by ctDNA, which can be detected earlier than clinical response.

Conclusion: In this study, we demonstrated that ctDNA is a prognostic marker for evaluating treatment response and predicting recurrence in advanced NSCLC. Further investigations with larger cohort and uniformed patient background are still needed to validate our findings.

Keywords: circulating tumor DNA, non-small-cell lung cancer, prognosis

\section{Introduction}

Non-small-cell lung cancer (NSCLC) has one of the highest mortality rates among all cancer types. ${ }^{1}$ The advance of genomic research and technology largely improves the development of precision medicine and further benefits the clinical outcomes of NSCLC patients. ${ }^{2,3}$ To profile the genomic alterations, tissues from tumor lesion is usually used by resection for molecular analysis. However, in some cases, tissue biopsy is hard to obtain due to logistic challenge or patient's body condition. Tissue samples may also fail to fully reflect the overall gene aberrances owing to the spatial and temporal tumor heterogeneity. ${ }^{4}$ In contrast, circulating tumor DNA (ctDNA), a noninvasive tumor marker separated from blood plasma, becomes an alternative biopsy to tissue sample..$^{5}$ ctDNA is also approved to be a good marker for treatment efficacy evaluation due to its rapid clearance from plasma.,

ctDNA is tumor-derived fragmented DNA released to plasma due to apoptosis and necrosis of tumor cells. The potential application value of ctDNA consists of diagnosis, acquired resistance mutation identification, targeted therapy guidance, and prognostic assessment. ${ }^{8,9}$ ctDNA has been validated as a predictive and prognostic marker to monitor treatment. In an early-stage breast cancer cohort study, detectable 
ctDNA at the first follow-up visit was associated with poor prognosis. ${ }^{10}$ In a colorectal cancer study, analysis of ctDNA status at the first follow-up visit after surgical resection suggested that the 3-year recurrence-free survival of ctDNApositive group was $0 \%$ and that of the ctDNA-negative group was $90 \% .^{11}$ More works are needed to further explore the predictive and prognostic value of ctDNA.

In this study, we sequenced the ctDNA from liquid biopsies from a cohort of Chinese advanced NSCLC patients. We analyzed the correlation of progression-free survival (PFS) and patient clinical characteristics at baseline. We evaluated the prognostic value of ctDNA by Kaplan-Meier analysis. We also presented two cases to illustrate the value of predicting disease progression by ctDNA, which can be detected earlier than radiographic imaging.

\section{Patients and methods}

\section{Patient information}

The entire study was approved by the institutional review board of Ningbo No. 2 Hospital. All patients provided written informed consent for this study.

\section{Preparation of plasma cell-free DNA}

Plasma cell-free DNA was isolated as described earlier. ${ }^{12}$ Ten milliliter of peripheral blood was collected and circulating cell-free DNA was recovered from plasma using the QIAamp Circulating Nucleic Acid kit by Qiagen (Valencia, CA, USA) following the manufacturer's instructions.

\section{Next-generation sequencing (NGS) library preparation and sequencing}

NGS library was prepared as described earlier. ${ }^{12}$ DNA shearing was performed using Covaris M220 (Covaris, Inc., Woburn, MA, USA), followed by end repair, phosphorylation, and adaptor ligation. Fragments of size 200-400 bp were selected by beads (Agencourt AMPure XP Kit, Beckman Coulter, Brea, CA, USA), followed by hybridization with capture probes baits, hybrid selection with magnetic beads, and PCR amplification. DNA quality and size were evaluated by high-sensitivity DNA assay using a bioanalyzer. Indexed samples were sequenced on a NextSeq 500 (Illumina, Inc., San Diego, CA, USA) with pair-end reads.

\section{Sequencing data analysis}

The sequencing data in the FASTQ format were mapped to the human genome (hg19) using BWA aligner 0.7.10. Local alignment optimization, variant calling, and annotation were performed using GATK 3.2, MuTect, and VarScan, respectively. DNA translocation analysis was performed using both Tophat 2 and Factera 1.4.3. Gene-level copy number variation was assessed using a $t$-statistic after normalizing reads depth at each region by total reads number and region size, and correcting GC-bias using a LOESS algorithm.

\section{Statistical analysis}

In the subcohort survival analysis, we used Kaplan-Meier analysis to estimate the survival functions and log-rank test to determine the difference of survival curve between groups. Multivariate analysis was performed using the Cox proportional hazards model, and all these analyses were conducted in $R$ (Version 3.3.3) with survival package (Version 2.41-3). Age difference between subgroups (with or without brain metastasis) was calculated with Student's $t$-test; a two-sided $p$-value of $<0.05$ was considered statistically significant.

\section{Results}

\section{Patient characteristics}

A total of 34 NSCLC patients at Stage IV were enrolled in this study. The median age was 63 years, ranging from 33 to 82 years old. Among them, 16 patients were female and 18 were male. Six patients had a history of smoking and 16 patients were nonsmokers, while 12 were unknown. Thirty-one cases were diagnosed as lung adenocarcinoma (LUAD); three cases were lung squamous cell carcinoma (LUSC). Seventeen patients had received surgery before and 14 patients did not receive surgery before; the past operation history of three patients were unknown. Twenty-five patients experienced past chemotherapy, while the other nine patients did not. Twenty-one patients received NGS-guided treatment and 12 patients did not follow NGS-guided therapy; data for the other one patient was unknown. Baseline of patient clinical characteristics is summarized in Table 1.

\section{Overview of the mutation spectrum revealed by NGS}

Liquid biopsies from all the 34 patients were performed with capture-based targeted ultradeep sequencing. We used a panel consisting of 168 genes, spanning 160 kilobases of human genomic regions. This ctDNA panel has been validated as described. ${ }^{12}$ We achieved a mean coverage depth of $12,810 \times$ across targeted regions. The corresponding white blood cells were used as a baseline to differentiate germline mutations.

A total of 96 mutations were identified from plasma ctDNA of the 34 patients at baseline. It included 68 singlenucleotide variants, 5 insertions and deletions, 15 copy-number 
Table I Summary of baseline patient characteristics

\begin{tabular}{|c|c|c|}
\hline $\begin{array}{l}\text { Patient } \\
\text { characterist }\end{array}$ & $\mathbf{n}$ & $\%$ \\
\hline Total & 34 & \\
\hline \multicolumn{3}{|l|}{ Gender } \\
\hline Female & 16 & 47.1 \\
\hline Male & 18 & 52.9 \\
\hline \multicolumn{3}{|l|}{ Age (years) } \\
\hline Median & 63 & - \\
\hline Range & $33-82$ & - \\
\hline \multicolumn{3}{|c|}{ Smoking history } \\
\hline Yes & 6 & 17.6 \\
\hline No & 16 & 47.1 \\
\hline Unknown & 12 & 35.3 \\
\hline \multicolumn{3}{|c|}{ Histological types } \\
\hline LUAD & 31 & 91.2 \\
\hline LUSC & 3 & 8.8 \\
\hline \multicolumn{3}{|l|}{ Past surgery } \\
\hline Yes & 17 & 50.0 \\
\hline No & 14 & 41.2 \\
\hline Unknown & 3 & 8.8 \\
\hline \multicolumn{3}{|c|}{ Past chemotherapy } \\
\hline Yes & 25 & 73.5 \\
\hline No & 9 & 26.5 \\
\hline \multicolumn{3}{|c|}{ NGS-guided treatment } \\
\hline Yes & 21 & 61.8 \\
\hline No & 12 & 35.3 \\
\hline Unknown & I & 2.9 \\
\hline
\end{tabular}

Note: “-” indicates not applicable.

Abbreviations: LUAD, lung adenocarcinoma; LUSC, lung squamous cell carcinoma; NGS, next-generation sequencing.

amplifications, 1 fusion, 6 frame-shifts, and 1 splicing site. We identified mutations from 79.4\% (27/34) patients, and the remaining 7 (20.6\%) patients had no mutation identified from this panel. The mutation spectrum of the cohort is shown in Figure 1A.

The most frequently mutated gene in this cohort was $E G F R$, accounting for $33.3 \%$ of all variants identified and occurring in $52.9 \%(18 / 34)$ patients. TP53 was the second most highly ranking mutations from the sequencing results, detected in $14.6 \%$ patients. We also identified several other oncogenic driver mutations, such as KRAS G12C and Q61K (2/34), ROS1 fusion (1/34), MET amplification (1/34), and $E R B B 220$ ins (1/34). Of the 18 EGFR-mutated patients, $6(33.3 \%)$ harbored EGFR tyrosine kinase inhibitors (TKIs)sensitizing mutations and 12 (66.7\%) harbored TKI-resistant mutations. Six patients were detected with first-generation EGFR-TKI-sensitizing mutation (one G719A, one exon 18del, one $19 \mathrm{del}$, and three L858R). Two patients harbored firstgeneration TKI-resistant mutations (one T790M + L858R and one T790M + 19del), and one patient had the thirdgeneration TKI-resistant mutation (in trans T790M + C797S; Figure 1B).

\section{Brain metastasis was correlated with PFS and age}

Of the 34 NSCLC patients, we followed up 21 patients who received NGS-guided targeted therapy after baseline ctDNA sequencing and collected their clinical information. We analyzed the correlation of prognosis and patient baseline characteristics in this subcohort. First, we explored the association of brain metastasis and PFS by Kaplan-Meier plot. The median PFS (mPFS) of patients with brain metastasis was shorter than patients without brain metastasis (101.5 days vs 144 days, $p=0.072$; Figure 2A). This suggested that brain metastasis in NSCLC was usually associated with inferior PFS. Next, we performed the relationship analysis of brain metastasis with age. Our data revealed a significant trend for younger NSCLC patients who were more prone to experience brain metastasis than older people (median age $=49.5$ year old vs 64 year old, $p=0.03$; Figure 2B).

\section{The association of baseline max allelic fraction (maxAF) and PFS}

maxAF was defined as the maximum somatic AF detected in a biopsy. A Cox proportional hazard model indicated that maxAF was correlated with PFS in the 21-patient subcohort $(p=0.03)$. We also performed Kaplan-Meier analysis and divided the subcohort into two groups: maxAF high and maxAF low. No significant mPFS difference can be observed between them. The Kaplan-Meier plot conclusion was similar when we divided the subcohort into three groups: maxAF high, maxAF medium, and maxAF low (Figure 3A and B). Next, we investigated the correlation of maxAF and PFS in osimertinib treatment group. For 10 patients who received following osimertinib treatment due to the positive of $E G F R$ T790M, we divided them into three groups according to different maxAF level. The result showed significant difference among them and maxAF was inversely correlated with PFS statistically ( $p=0.029$; Figure $3 \mathrm{C})$.

\section{ctDNA increase predicts potential of progressive disease}

Plasma ctDNA sequencing can be used as a noninvasive tool to detect cancer mutations. The predictive value of ctDNA has been investigated in different cancer types. In this study, we focused on two patients and performed longitudinal sequencing to further confirm the predictive value by monitoring the ctDNA change. For patient 1 , ctDNA sequencing in stable disease (SD) stage revealed a newly acquired resistance mutation EGFR T790M accompanied with increasing $E G F R$-sensitizing mutation L858R 
A

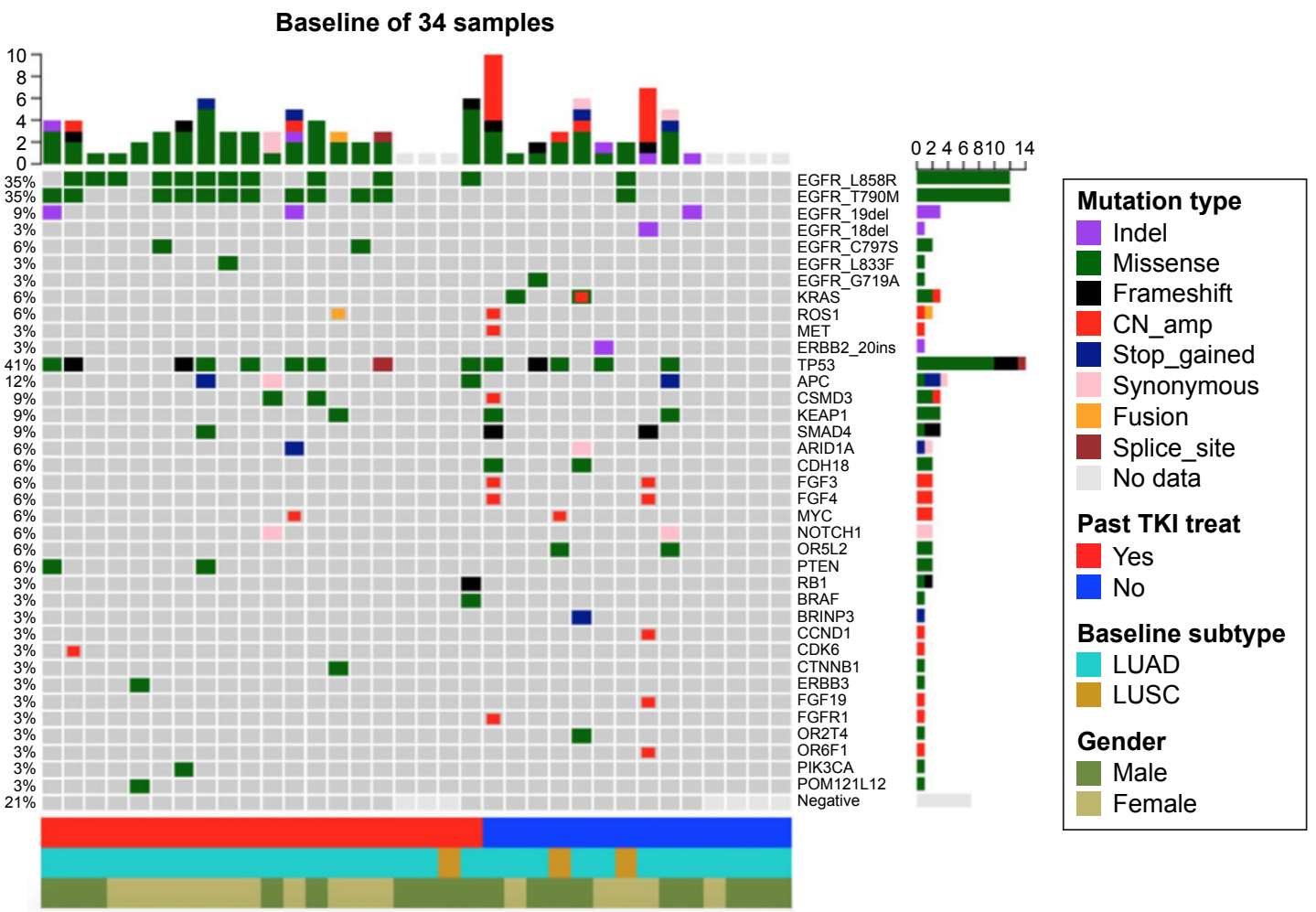

B

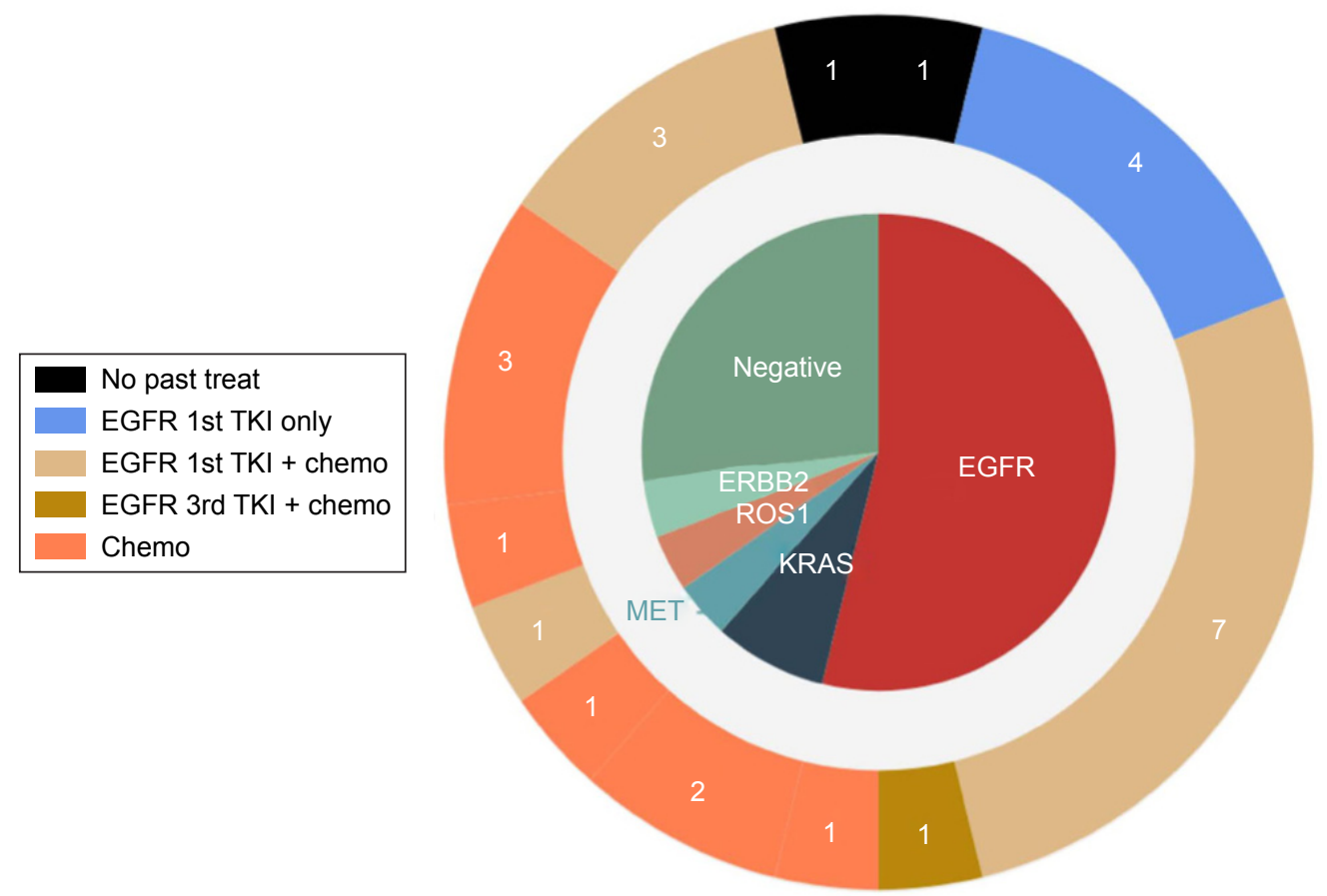

Figure I Mutations identified in baseline plasma ctDNA of the cohort consisting of 34 NSCLC patients.

Notes: (A) Mutation spectrum oncoprint of baseline ctDNA. The picture also illustrated the treatment history and histological subtype. (B) Schematic diagram of identified oncogenic mutations by NGS. The number in outer circle indicates the number of patients who received corresponding past treatment.

Abbreviations: ctDNA, circulating tumor DNA; NGS, next-generation sequencing; NSCLC, non-small-cell lung cancer; TKI, tyrosine kinase inhibitor.

compared to baseline. This contributes to the first-generation TKI erlotinib resistance and later clinical progression disease (PD), with a lead time of 58 days (Figure 4A). Patient 2 was detected with increased EGFR L858R during the SD stage under osimertinib treatment, suggesting the emergency of underlying resistances that cannot be revealed by the NGS at that time. He developed with PD after 22 days and following sequencing confirmed newly acquired ERBB2 
A

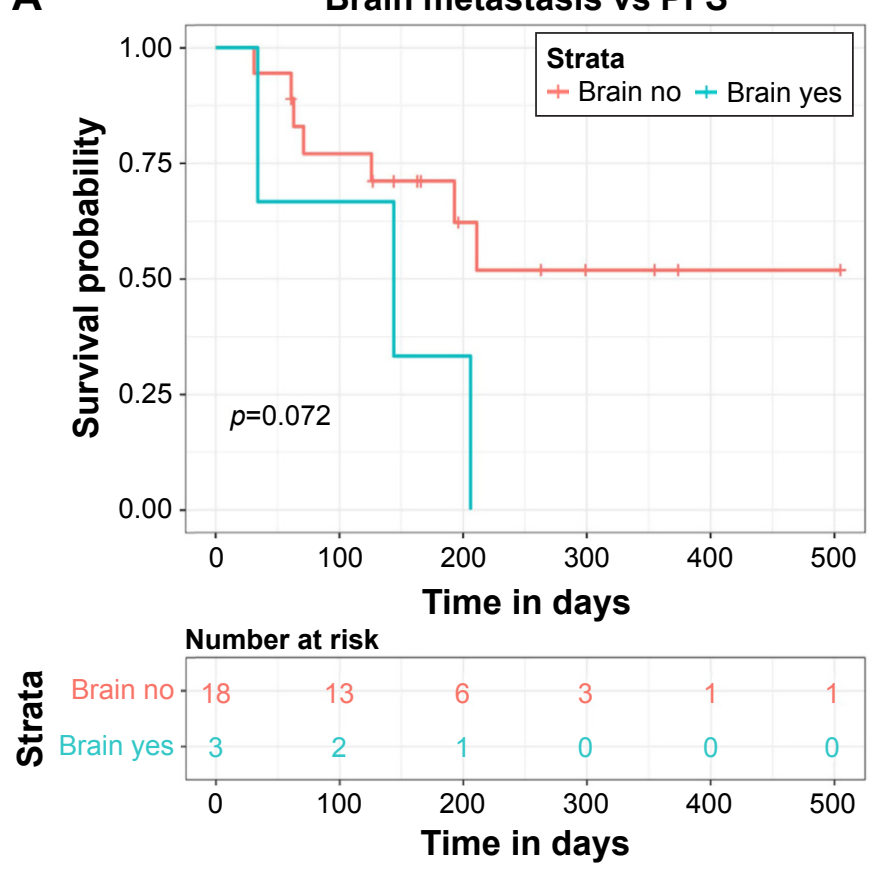

B

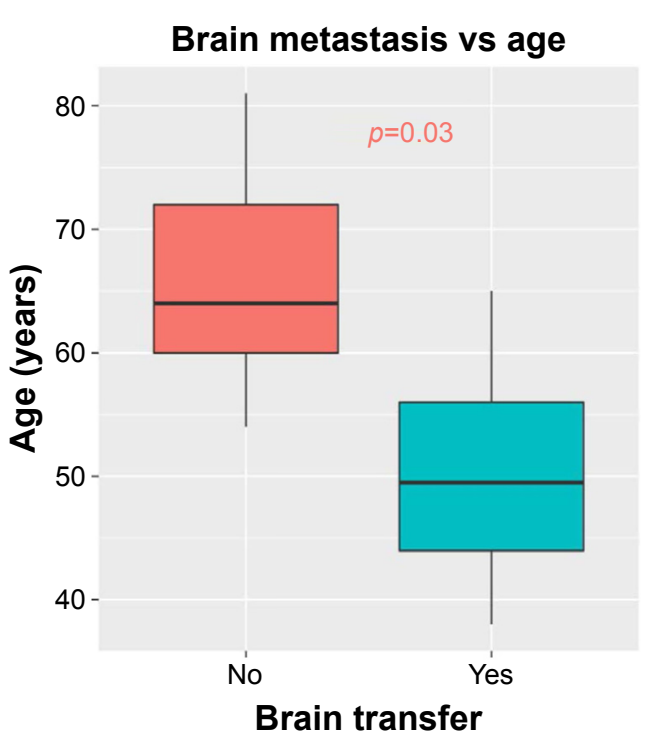

Figure 2 Kaplan-Meier analysis of the correlation between baseline patient characteristics and PFS. Notes: (A) Patients with brain metastasis associated with a shorter PFS. (B) Patients with brain metastasis correlated with younger age. Abbreviation: PFS, progression-free survival.

and EGFR amplification, which could lead to osimertinib resistance. PIK3CA E542K, a potential resistance mutation to third-generation EGFR-TKI, was also detected in SD stage of osimertinib treatment in patient 2. It may also be a predictor of following $\mathrm{PD}$ and contribute to the final disease progression (Figure 4B).

\section{Discussion}

In this study, we performed ultradeep targeted capture-based sequencing in plasma ctDNA samples from a cohort of 34 Chinese advanced NSCLC patients. Our study confirmed and further extended the notion that liquid biopsy detection using high-throughput sequencing technologies was a valuable tool to predict the treatment response and prognosis. We demonstrated a strong association between brain metastasis at baseline and PFS or age. We also investigated the inverse relationship of baseline maxAF and PFS in osimertinib treatment subcohort.

Plasma ctDNA has been approved to be a noninvasive tool and real-time biomarker to provide prognostic information, and most of these studies were focusing on treatmentnaive patients. In this study, we extended our investigation scale to all NSCLC patients irrespective of whether they had undergone previous treatment or note. We performed prognosis analysis between groups based on different baseline ctDNA parameters. Pecuchet et $\mathrm{al}^{13}$ reported that baseline
ctDNA positivity was independent of PFS in a NSCLC cohort, and other researchers reported that the presence of ctDNA was associated with a shorter PFS. ${ }^{14}$ Our study illustrated that higher maxAF at baseline ctDNA detection was associated with poor prognosis in osimertinib treatment group. But in the whole cohort with diverse previous treatment histories, there was no correlation of baseline maxAF and PFS. The inconsistent observations of these studies may attribute to the limited patient number and diverse patient clinical characteristics. Studies including larger cohort and uniformed patient background are needed to further investigate the prognostic value of ctDNA. Moreover, we foresee that a broader range of studies will be conducted in various cohorts with complicated disease status to further extend our knowledge of ctDNA value for cancer prognostic.

Radiological examination such as CT scan can only detect residue disease that is presented as tumor bulk. Plasma ctDNA is used not only as a biomarker of tumor occurrence but also a predictor of tumor recurrence by dynamically monitoring the clonal changes. ${ }^{13,15}$ Moreover, the tumor cells needed for ctDNA detected is much lower than that for CT scan imaging. ctDNA sequencing can also reach a much higher sensitivity and specificity than CT scan. ${ }^{11}$ Several previous studies have reported the strong correlation of ctDNA dynamics and tumor recurrence. In a breast cancer patient cohort, ctDNA provided the earliest indication of treatment response, as well 
A
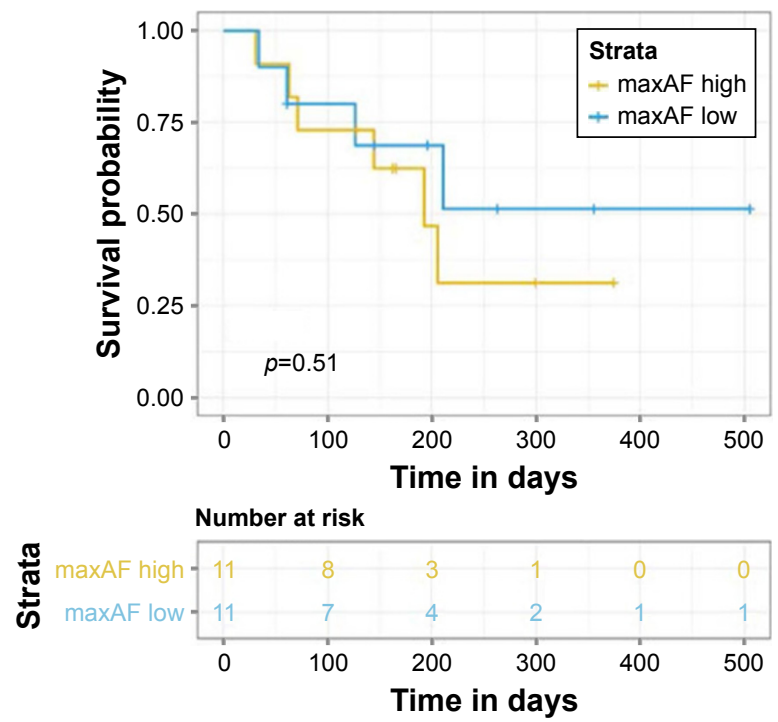

B
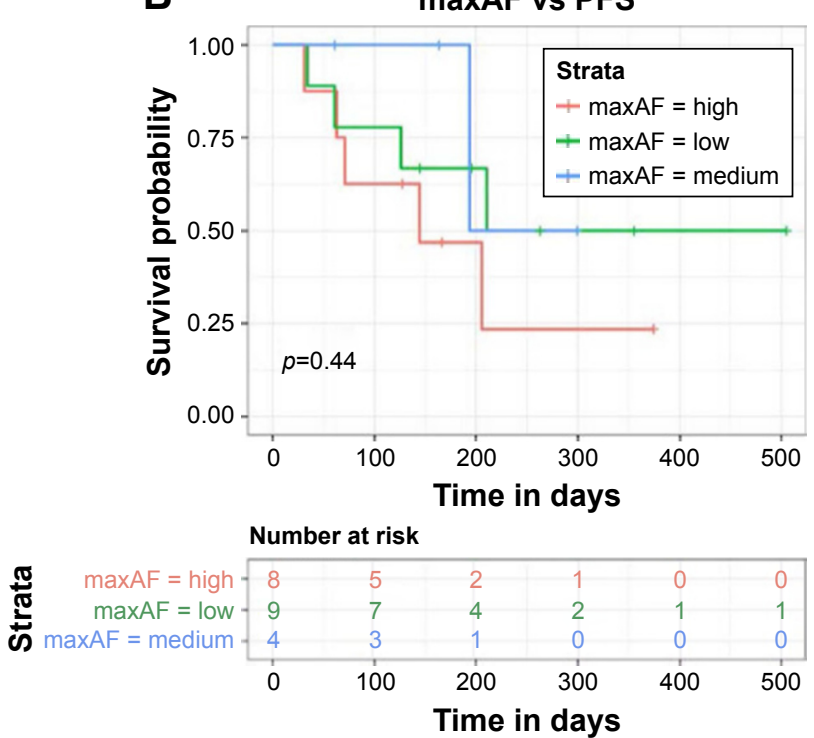

C maxAF vs PFS (osimertinib group)
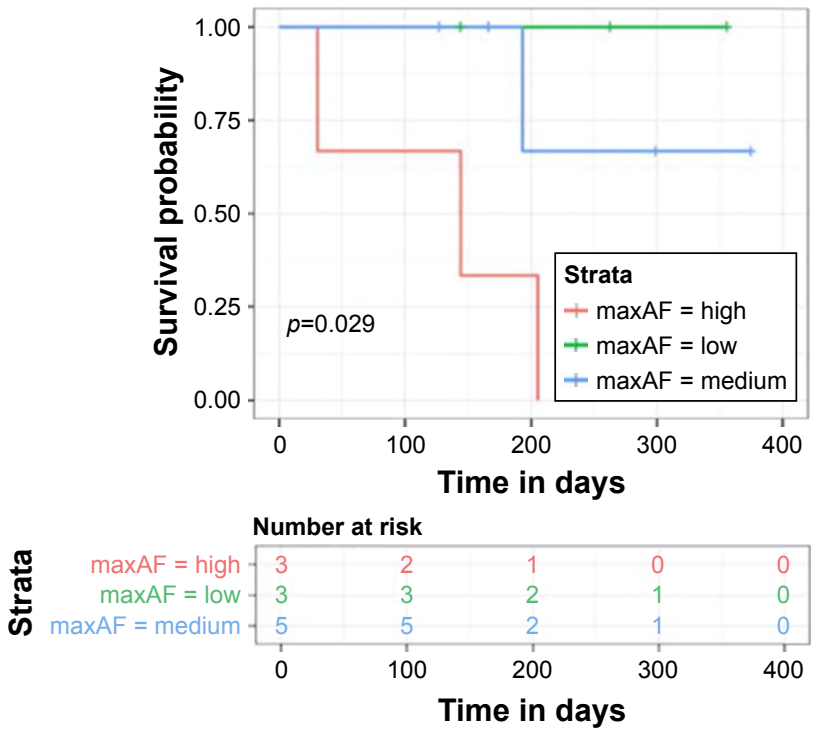

Figure 3 Correlation of baseline ctDNA maxAF and PFS.

Notes: (A) The association of ctDNA maxAF and PFS when the cohort was divided into two groups based on different maxAF. (B) Correlation of maxAF and PFS when 34 patients were divided into three groups based on maxAF level. (C) The relationship of maxAF and PFS in osimertinib-treated subgroup.

Abbreviations: ctDNA, circulating tumor DNA; maxAF, max allelic fraction; PFS, progression-free survival.

as the earliest hint of disease relapse compared to imaging and other biomarkers such as CA15-3. ${ }^{16}$ Stage II colon cancer patients who were ctDNA positive after operation were reported to be at extremely high risk of radiologic recurrence. ${ }^{11}$ In this study, we presented two patients with ctDNA monitoring during the treatment process. In patient 1, NGS detected the classic erlotinib-resistant mutation EGFR T790M 58 days earlier than clinical PD revealed by radiographic imaging. In patient 2 , the increasing allelic fraction of L858R and newly acquired PIK3CA E542K both indicated the emergency of osimertinib resistance, 22 days prior to the clinical PD. Following NGS further confirmed the osimertinib-resistant mechanism: PIK3CA E542K accompanied with $E G F R$ amplification and $E R B B 2$ amplification. It highlighted the notion that liquid biopsy can be served as a valuable tool to monitor and predict the clinical response earlier than radiographic imaging.

\section{Conclusion}

We demonstrated that plasma ctDNA can be feasible and effective in prognosis and prediction of treatment response and disease progression. This study illustrated the significant 

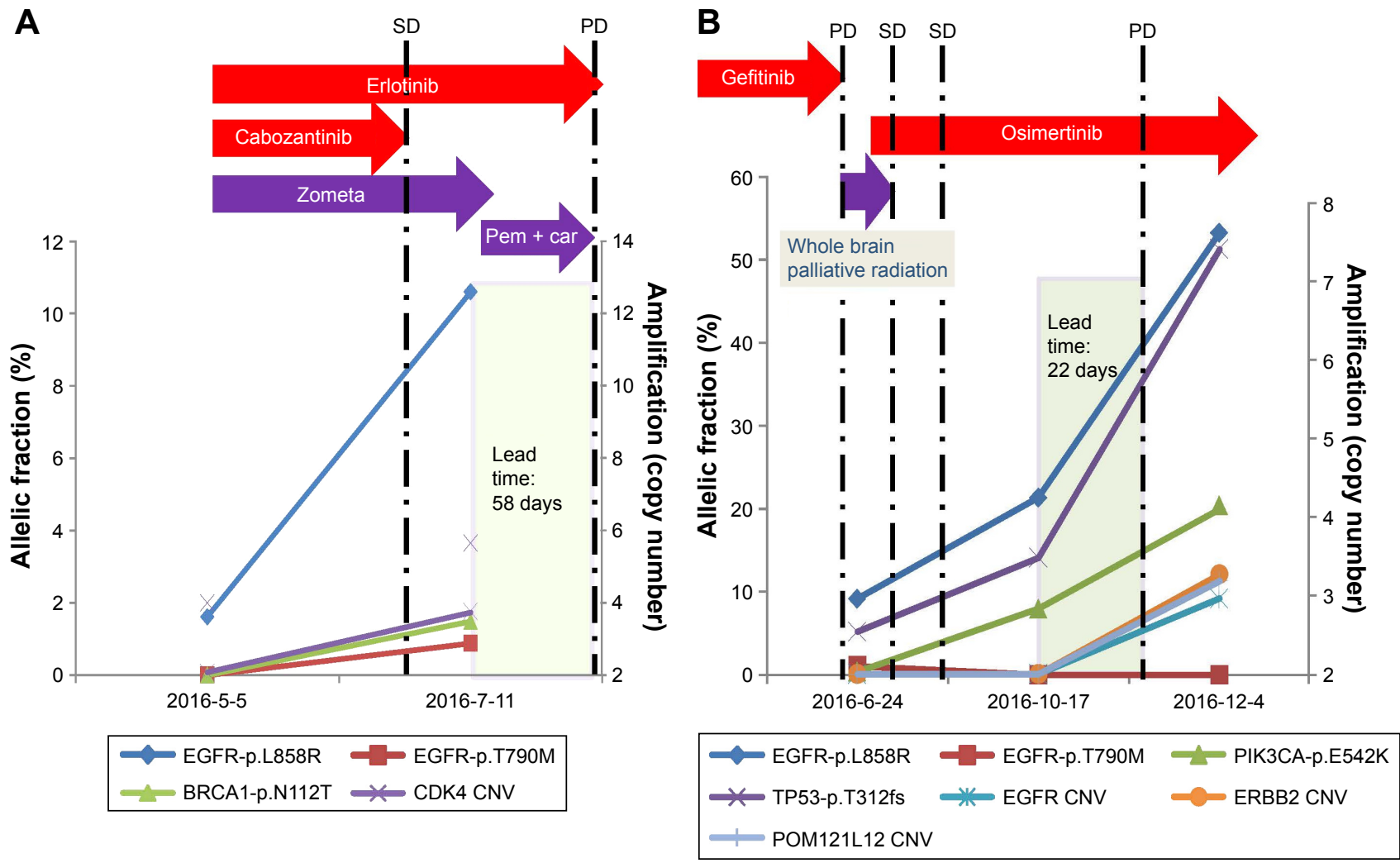

Figure 4 Two cases are presented to illustrate the value of ctDNA in predicting disease progression earlier than radiographic imaging. Notes: (A) Patient I; (B) patient 2.

Abbreviations: Car, carboplatin; ctDNA, circulating tumor DNA; SD, stable disease; PD, progression disease; Pem, pemetrexed.

value of the noninvasive tool in investigating and monitoring the genetic landscapes of tumors, which had great clinical implications for identifying and guiding treatment strategy.

\section{Disclosure}

The authors report no conflicts of interest in this work.

\section{References}

1. Jemal A, Bray F, Center MM, Ferlay J, Ward E, Forman D. Global cancer statistics. CA Cancer J Clin. 2011;61(2):69-90.

2. Swanton C, Govindan R. Clinical implications of genomic discoveries in lung cancer. $N$ Engl J Med. 2016;374(19):1864-1873.

3. Tursz T, Andre F, Lazar V, Lacroix L, Soria JC. Implications of personalized medicine - perspective from a cancer center. Nat Rev Clin Oncol. 2011;8(3):177-183.

4. Gerlinger M, Rowan AJ, Horswell S, et al. Intratumor heterogeneity and branched evolution revealed by multiregion sequencing. $N$ Engl $J$ Med. 2012;366(10):883-892.

5. Bidard FC, Weigelt B, Reis-Filho JS. Going with the flow: from circulating tumor cells to DNA. Sci Transl Med. 2013;5(207):207ps214.

6. Lo YM, Zhang J, Leung TN, Lau TK, Chang AM, Hjelm NM. Rapid clearance of fetal DNA from maternal plasma. Am J Hum Genet. 1999; 64(1):218-224.

7. Diehl F, Schmidt K, Choti MA, et al. Circulating mutant DNA to assess tumor dynamics. Nat Med. 2008;14(9):985-990.
8. Crowley E, Di Nicolantonio F, Loupakis F, Bardelli A. Liquid biopsy: monitoring cancer-genetics in the blood. Nat Rev Clin Oncol. 2013; 10(8):472-484.

9. Aparicio S, Caldas C. The implications of clonal genome evolution for cancer medicine. N Engl J Med. 2013;368(9):842-851.

10. Garcia-Murillas I, Schiavon G, Weigelt B, et al. Mutation tracking in circulating tumor DNA predicts relapse in early breast cancer. $S c i$ Transl Med. 2015;7(302):302ra133.

11. Tie J, Wang Y, Tomasetti C, et al. Circulating tumor DNA analysis detects minimal residual disease and predicts recurrence in patients with stage II colon cancer. Sci Transl Med. 2016;8(346):346ra392.

12. Mao X, Zhang Z, Zheng X, et al. Capture-based targeted ultradeep sequencing in paired tissue and plasma samples demonstrates differential subclonal ctDNA-releasing capability in advanced lung cancer. J Thorac Oncol. 2017;12(4):663-672.

13. Pecuchet N, Zonta E, Didelot A, et al. Base-position error rate analysis of next-generation sequencing applied to circulating tumor DNA in non-small cell lung cancer: a prospective study. PLoS Med. 2016; 13(12):e1002199.

14. Pietrasz D, Pecuchet N, Garlan F, et al. Plasma circulating tumor DNA in pancreatic cancer patients is a prognostic marker. Clin Cancer Res. 2017;23(1):116-123.

15. Wan JCM, Massie C, Garcia-Corbacho J, et al. Liquid biopsies come of age: towards implementation of circulating tumour DNA. Nat Rev Cancer. 2017;17(4):223-238.

16. Dawson SJ, Tsui DW, Murtaza M, et al. Analysis of circulating tumor DNA to monitor metastatic breast cancer. $N$ Engl J Med. 2013;368(13):1199-1209. 


\section{Publish your work in this journal}

OncoTargets and Therapy is an international, peer-reviewed, open access journal focusing on the pathological basis of all cancers, potential targets for therapy and treatment protocols employed to improve the management of cancer patients. The journal also focuses on the impact of management programs and new therapeutic agents and protocols on

patient perspectives such as quality of life, adherence and satisfaction. The manuscript management system is completely online and includes a very quick and fair peer-review system, which is all easy to use. Visit http://www.dovepress.com/testimonials.php to read real quotes from published authors.

Submit your manuscript here: http://www.dovepress.com/oncotargets-and-therapy-journal 